\title{
Nueva estrategia para el manejo de los lípidos en prevención cardiovascular
}

\author{
New strategy for the management of lipids in cardiovascular prevention
}

Juán Franco

Juan Franco. Nueva estrategia para el manejo de los lípidos en prevención cardiovascular. Evid Act Pract Ambul. Ene-Mar 2014;17(1):2-3.

Descripción del tamaño del efecto y la precisión de la estimación

El Programa Nacional de Educación sobre Colesterol (National Cholesterol Education Program, NCEP), dependiente del Instituto Nacional del Corazón, Pulmón y Sangre (National Heart, Lung and Blood Institute, NHLBI) de EE.UU. había publicado en $2002^{1}$ el Reporte del Panel de Expertos para el Tratamiento del Colesterol en Adultos (Adult Treatment Panel III, ATP III) con una ulterior modificación en $2004^{2}$. En dichos documentos habían establecido varios puntos clave:

- Metas de colesterol LDL (LDL-C: liproproteínas de baja densidad) como principal objetivo terapéutico para la reducción del riesgo cardiovascular.

- Clasificación de los individuos en diferentes subgrupos de riesgo, cada uno con diferentes objetivos de LDL-C: LDL-C más bajos ante enfermedad cardiovascular (ECV) manifiesta (prevención secundaria) o diabetes (como "equivalentes de ECV") y diferentes metas de acuerdo al riesgo calculado a través del puntaje de Framingham.

- Uso de tratamientos complementarios para alcanzar las diferentes metas de LDL-C (ver tabla 1).

Tabla 1: metas de LDL-C de acuerdo al perfil individual de riesgo cardiovascular propuestas por el Consenso ATP III (2004).

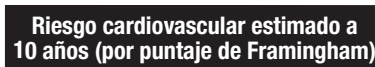

ECV manifiesta o RCV estimado mayor a 20\% Menor a $100 \mathrm{mg} / \mathrm{dL}$ (u opcionalmente $70 \mathrm{mg} / \mathrm{dL}$ ) \begin{tabular}{l|r}
\hline RCV $10-20 \%$ o 2 FRCV con RCV menor a $10 \%$ & Menor a $130 \mathrm{mg} / \mathrm{dL}$ \\
\hline
\end{tabular}

Ninguno o un FRCV Menor a $160 \mathrm{mg} / \mathrm{dL}$

ECV: enfermedad cardiovascular. RCV: riesgo cardiovascular. FRCV: factores de riesgo cardiovascular. *Para pacientes de muy alto riesgo (pacientes con diabetes y enfermedad cardiovascular manifiesta).

Guía del Colegio Norteamericano de Cardiología y de la Asociación Norteamericana del Corazón (2013)

La nueva guía que hemos resumido a continuación en este número de EVIDENCIA, desarrollada originalmente en conjunto por el NHLBI, el Colegio Americano de Cardiología (American College of Cardiology, ACC) y la Asociación Americana del Corazón (American Heart Association, AHA) presenta un nuevo paradigma en el tratamiento del colesterol como estrategia para la reducción del riesgo cardiovascular. Los puntos clave propuestos en esta nueva guía son:

- La eliminación de las metas de LDL-C específicas.

- El uso de dosis fijas de estatinas de acuerdo al riesgo cardiovascular estimado.

- La consideración de poblaciones especiales para quienes el uso de estatinas acarrea un beneficio incierto.

- La propuesta de una nueva calculadora de riesgo cardiovascular ajustada a la población estadounidense (Pooled Cohorts Equations) ${ }^{3}$ nutrida de datos de varios estudios longitudinales. - Dejar sin efecto las recomendaciones de uso de otros hipolipemiantes. Estas recomendaciones se ajustan con más fidelidad a los resultados que arrojaron los ensayos clínicos incluidos en la revisión sistemática que respalda a esta guía. A partir del análisis del riesgo cardiovascular de los participantes de dichos ensayos, los autores de este consenso llegaron a la conclusión de que un umbral de $7,5 \%$ de riesgo de desarrollo de ECV a diez años define a una población con altas probabilidades de obtener un beneficio neto del uso de estatinas, necesitándose tratar a 50 o 100 personas durante diez años para que una de ellas evite un evento adicional. (NNT entre 50 y 100), aún con- siderando el bajo riego de desarrollo de diabetes atribuido al uso de estatinas (Número Necesario a Dañar: NND mayor a 100).

Luego de evaluar sistemáticamente esta guía con la herramienta $\mathrm{AGREE}^{4}$, que permite calificar su calidad, pudimos constatar que sus autores tuvieron escasa consideración respecto de las perspectivas individuales del paciente, que no discutieron los procesos organizativos y barreras para su aplicación, y que brindaron escasa información sobre los costos asociados a esta estrategia de tratamiento. Por otra parte, gran parte del panel explicitó importantes potenciales conflictos de intereses por su conexión con la industria farmacéutica.

\section{Repercusiones}

Las principales revistas de alto impacto publicaron comentarios editoriales sobre las nuevas guías ${ }^{5,6,7}$. Entre sus puntos destacaron:

- El sinceramiento sobre la nula evidencia para el uso de metas específicas de LDL-C y respecto del empleo de hipolipemiantes no estatínicos para alcanzarlas.

- El menor énfasis en el uso de marcadores con incierto poder predictivo de riesgo cardiovascular ${ }^{8}$ (proteína $\mathrm{C}$ reactiva, puntaje de calcio, etc.)

- El enfoque conservador propuesto para el abordaje de las poblaciones en quienes la evidencia es escasa o conflictiva (menores de 45 años y mayores de 75).

Las principales críticas apuntaron hacia:

- La fijación de umbrales de riesgo cardiovascular arbitrarios para la indicación de altas dosis de estatinas.

- La sobreestimación del riesgo cardiovascular a través de la nueva calculadora propuesta, aspecto reportado por el mismo Grupo de Trabajo que la diseñó a través de su validación en distintas cohortes

- La existencia de conflictos de intereses con la industria farmacéutica en ocho de los 15 panelistas.

La investigación de Pencina y col. ${ }^{9}$, basada en la subpoblación estadounidense de 40 a 75 años de edad que participó de la Encuesta del Examen de Salud Nutricional (National Health and Nutrition Examination Surveys, NHANES) estimó a que proporción de la población de EE.UU. se le recomendaría el uso de estatinas, comparando los criterios de prescripción de la guía ATP III (2004) con los de la nueva guía de la ACC/AHA (2013). Este trabajo arrojó los siguientes resultados:

- Un $42 \%$ de la población seleccionada de la NHANES recibiría estatinas según la guía ATP III y un $56,6 \%$ de acuerdo a la ACC/AHA (2013), lo que, extrapolado a la población estadounidense, representaría 12,8 millones de nuevos pacientes que comenzarían a utilizar estatinas.

- Este incremento se produciría en todas las categorías de riesgo cardiovascular, pero fundamentalmente en aquellos pacientes sin enfermedad cardiovascular ni diabetes (prevención primaria) con un riesgo estimado de eventos a diez años mayor a $7.5 \%$ (8.1 millones de nuevos pacientes).

- Este efecto sobre la prevención primaria sería atribuible principalmente al descenso de umbral de riesgo cardiovascular para la prescripción de estatinas. Vale destacar que en la población de 60 a 75 años, el uso de la nueva calculadora (regla de predicción clínica) reclasificaría a casi el $30 \%$ de los pacientes con un riesgo igual o mayor a $7,5 \%$.

El estudio de Kavousi y col. ${ }^{10}$ comparó la aplicación de la guía ATP III, la guía de la Sociedad Europea de Cardiología / Sociedad 
Europea de Aterosclerosis (European Society of Cardiology / European Atherosclerosis Society, ESC/EAS) y la nueva guía ACC/AHA 2013 en una cohorte holandesa de pacientes entre 55 y 75 años y llegó a las siguientes conclusiones:

- El $96,4 \%$ de los hombres y el $65.8 \%$ de las mujeres de esta cohorte tendrían indicación de estatinas según la guía ACC/AHA en comparación con el $66,1 \%$ y el $39,1 \%$ a quienes se les prescribiría estos fármacos siguiendo los criterios de la ESC/EAS.

- Las tres calculadoras (Framingham"1, Pooled Cohort Equations ${ }^{12}, \mathrm{SCORE}^{13}$ ) que fueron utilizadas en las guías de práctica clínica tuvieron pobre calibración en relación con los eventos observados y sobreestimaron el riesgo cardiovascular a diez años en todas las categorías de riesgo. Por ejemplo, el $88 \%$ de los varones de ésta población fueron categorizados en el subgrupo que poseería un riesgo mayor a 10\% de desarrollar enfermedad cardiovascular a diez años según la Pooled Cohort Equations (promedio calculado 21,5\%), y sin embargo, sólo el $12,7 \%$ realmente desarrolló ECV. Usando la regla de predicción SCORE en la misma población de varones, el riesgo calculado promedio de muerte por ECV a diez años fue de $6,8 \%$, contra un realmente observado de $3,8 \%$.

Vale destacar que actualmente un grupo británico de trabajo está desarrollando una nueva guía para el tratamiento del colesterol en el Reino Unido (Instituto Nacional de Excelencia de Salud y Cuidados, National Institute for Health and Care Excellence, NICE) que incluiría puntos clave similares a la de la ACC/AHA 2013 (eliminación de metas de LDL-C, nueva calculadora de riesgo, etc.). El borrador ${ }^{14}$ de la misma está disponible en Internet para la sugerencia de modificaciones.

\section{Conclusiones}

La nueva guía ACC/AHA 2013 provee una nueva perspectiva sobre el uso de estatinas para la reducción del riesgo cardiovascular ya que el otrora importante foco en el descenso del LDL-C ha quedado relativizado junto a la valoración de otras variables vinculadas con el riesgo cardiovascular global.

Una limitación de vital importancia, que todavía no ha sido resuelta es la incertidumbre que todavía tenemos al aplicar éstas calculadoras a poblaciones latinoamericanas en las cuales no conocemos el grado de sobre o subestimación del riesgo cardiovascular ${ }^{15}$. Vale destacar que cada una de las reglas de predicción clínica mencionadas está respaldada por cohortes locales de EE.UU. y Europa; y que si bien ya existen proyectos en marcha ${ }^{\S}$ como el del equipo CESCAS ${ }^{16}$, actualmente no contamos con datos de seguimientos prolongados de América del Sur. Finalmente, y ante la incertidumbre de la aplicabilidad de estas guías en nuestra población, consideramos adecuado discutir la decisión con nuestros pacientes, complementándola con recursos informativos gráficos para la toma de decisiones compartidas como los disponibles en castellano en la página de la Mayo Clinic

(http://statindecisionaid.mayoclinic.org/?lang=es_es) ${ }^{19,20}$.Ver figura 1.

Figura 1: captura de pantalla de la Herramienta de la Clínica Mayo para la Toma de Decisiones compartidas respecto de la indicación de estatinas y aspirina en función del riesgo cardiovascular del paciente.

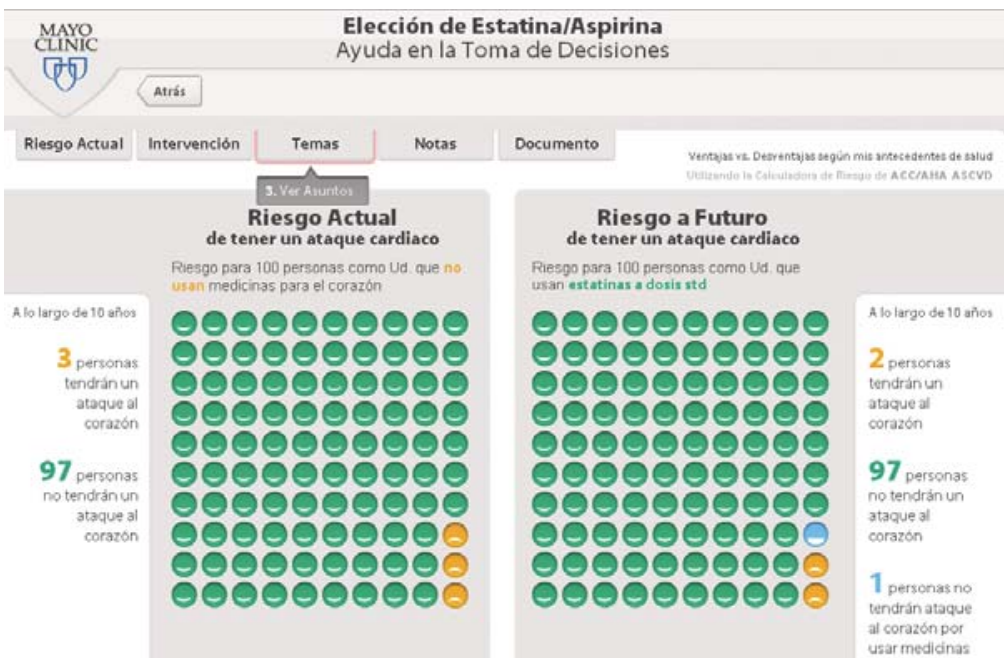

Referencias

1. National Cholesterol Education Program (NCEP) Expert Panel on Detection, Evaluation, and Treatment of High Blood Cholesterol in Adults (Adult Treatment Panel III). Third Report of the National Cholesterol Education Program (NCEP) Expert Panel on Detection, Evaluation, and Treatment of High Blood Cholesterol in Adults (Adult Treatment Panel III) final report. Circulation. 2002 Dec 17;106(25):3143-421.

2. Grundy SM, Cleeman JI, Merz CN, Brewer HB Jr, Clark LT, Hunninghake DB, Pastemak RC, Smith SC Jr, Stone NJ; Coordinating Committee of the National Cholesterol Education Program. Implications of recent clinical trials for the National Cholesterol Education Program Adult Treatment Panel III guidelines. Arterioscler Thromb Vasc Biol. 2004 Aug;24(8):e149-61

3. Goff DC Jr, Lloyd-Jones DM, 2013 ACC/AHA Guideline on the Assessment of Cardiovasall

Nov 12. pii: S0735-1097(13)06031-2. doi: 10.1016/j.j.jacc.2013.11.005. [Epub ahead of print]
4. The Agree Collaboration. AGREE Instrument Spanish version, 2001 http://www.agreecollaboration.org

5. loannidis JP. More than a billion people taking statins?: Potential implications of the new cardiovascular guidelines. JAMA. 2014 Feb 5;311(5):463-4. doi: 10.1001/jama.2013.284657.

6. Keaney JF Jit, Curfman GD, Jarcho JA. A pragmatic view of the new cholesterol treatment guidelines. N Engl J Med. 2014 Jan 16;370(3):275-8. doi: 10.1056/NEJMMms 1314569. Epub 2013 Nov 27.

7. Krumholz HM. Target cardiovascular risk rather than cholesterol concentration. BMJ. 2013 Nov 27;347:f7110. doi: 10.1136/bmiff7110.

8. Terrasa y col. Marcadores serológicos y clínicos en la predicción del riesgo cardiovascular: ¿nuevos factores de riesgo? Evid. Act Pract Ambul. 14 (1). 12-17.Ene.Mar.2011.

9. Pencina MJ1, Navar-Boggan AM, Application of new cholesterol guidelines to a population-based sample. N Engl J Med. 2014 Apr 10;370(15):1422-31. doi: 10.1056/NEJMoa1315665. Epub 2014 Mar 19.

10. Kavousi M1, Leening M.J2, Comparison of application of the ACC/AHA guidelines, Adult Treatment Panel III guidelines, and European Society of Cardiology guidelines for cardiovascular disease prevention in a European cohort. JAMA.

2014 Apr 9;311(14):1416-23. doi: 10.1001/jama.2014.2632.

11. D'Agostino RB Sr, Grundy S, Sullivan LM, Wilson P; CHD Risk Prediction Group. Validation of the Framingham coronary heart disease

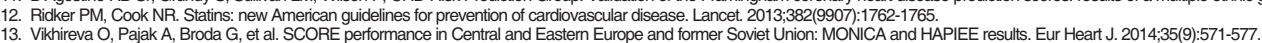
sultation. 12 Feb 2014. http://guidance.nice.org.uk/CG/WaveR/123.

15. Terasa S, Kopitowski K, Evaluación del riesgo cardiovascular global (segunda parte), Evid. actual. práct. ambul; 7(2): 52-55, mar-abr. 2004

16. Rubinstein A y col. Kstudio de detección y seardioviscular global (segunda parte), Evid. actual. práct. ambul, $7(2) .52-55$, mar.-abr. 2004 16. Rubinstein A y col. Estudio de detección y seguimimis
visor.php?cod_producto= 758 (útimo acceso 11/04/14).

17. Barani M, Kopitowski K, Toma de decisiones compartidas: centrando los cuidados médicos realmente en nuestros pacientes, Rev. Hosp. Ital. B. Aires (2004); 33(2): 60-64, jun. 2013

18. Mayo Clinic, Statin / Aspirin choice, decision aid http://statindecisionaid.mayoclinic org/ (último acceso 11/04/14)

19. Barani M, Kopitowski K, Toma de decisiones compartidas: centrando los cuidados médicos realmente en nuestros

$\S$ Recordamos que esto se refiere a que existe una probabilidad más alta que la aceptada de que ese hallazgo haya sido por azar. 